\title{
Potential Impact of Male Circumcision, Condom Use, and Microbicides on the Dynamics of HIV/AIDS
}

\author{
Felix Elias $^{1}$ and Jean M. Tchuenche ${ }^{2}$ \\ ${ }^{1}$ Dar es Salaam Institute of Technology, Dar es Salaam, Tanzania \\ ${ }^{2}$ Mathematics Department, University of Dar es Salaam, Dar es Salaam, Tanzania
}

Correspondence should be addressed to Felix Elias, felias77@yahoo.co.uk

Received 15 May 2012; Accepted 15 August 2012

Academic Editors: B. Foy and M. Santillán

Copyright (C) 2012 F. Elias and J. M. Tchuenche. This is an open access article distributed under the Creative Commons Attribution License, which permits unrestricted use, distribution, and reproduction in any medium, provided the original work is properly cited.

\begin{abstract}
HIV/AIDS remains an important global cause of morbidity and mortality. While medical male circumcision and condom and microbicides use hold great promise for helping to stem the tide of new HIV infections, theoretically providing further evidence of the potential long-term population-level benefit of their combined effects is viable. A deterministic sex-structured model is formulated, the expected lifetime disease reproductive output of an individual (or epidemiological birth) is determined, and the stability of steady states is investigated. To complement HIV treatment with antiretrovirals, which is not yet fully accessible to all those in need, microbicides and nontherapeutic measures such as male circumcision and condoms provide additional potential impact on curtailing the spread of HIV/AIDS.
\end{abstract}

\section{Introduction}

"Globally, HIV/AIDS has killed more than 25 million people, making it one of the most destructive epidemics in recorded history with sub-Saharan Africa carrying the highest burden of the disease. Since its emergence in 1981, HIV continues to be a significant social-economic and public health burden around the globe. Its main transmission route is heterosexual, representing up to $90 \%$ of all transmission." For the estimated 33.3 million (27.1-30.3 million) people living with HIV after nearly 30 years into a very complex epidemic, the gains are real but still fragile, even as the number of annual AIDS-related deaths worldwide has steadily decreased from the peak of 2.1 million [1.9-2.3 million] in 2004 to an estimated 1.8 million [1.6-2.0 million] in 2009 [1].

Various preventative and therapeutic measures have been embarked upon, aiming at combating one of the greatest pandemic in modern times. These include the use of lifesaving antiretroviral drugs (ARVs), condoms, HIV testing, mutual monogamy, counseling, and abstinence ( $\mathrm{WHO} /$ UNAIDS, 2005) [2, 3]. Various degrees of success of these controls have been limited to very few countries [4]. Consistent use of latex condoms which is advocated as a primary prevention for HIV infection [5, 6] has suffered from problems of stigma, myths and fertility decline which are key social issues in sub-Saharan Africa [4]. Although the use of antiretroviral drugs has led to significant reduction in HIV burden in many nations, their accessibility and efficient delivery in resource-poor nations remain low. These together with the absence of an anti-HIV vaccine, call for use of other affordable programs for controlling HIV spreading especially in nations with limited resources and inadequate public health services.

Male circumcision which provides only partial protection [7-13] has been shown to reduce the risk of men acquiring HIV through female-to-male transmission by $60 \%$ $[7,14]$, while microbicides are considered one of the most promising preventive interventions to emerge over the past decade $[15,16]$. Microbicides which are $60 \%$ effective in reducing the risk of acquiring HIV could prevent 3.7 million infections within 3 years $[17,18]$. In resourcelimited settings, most women do not have the social or economic power necessary to insist on condom use and fidelity or to abandon partnerships that put them at risk. Because men often decide whether or not to use a condom during intercourse, it is generally difficult or impossible for 
women to ensure consistent condom use by their partners. Microbicides therefore put the power of women's protection against HIV infection into their own hands as they do not require partners' cooperation [2]. Indeed, at the Fifth World AIDS Conference in Vienna, 2010, the CAPRISA 004 investigators reported a 39\% reduction in HIV incidence, providing a proof of concept for microbicides [19]. Condoms which contribute at preventing HIV transmission have been assessed [6], while the effect of jointly implementing male circumcision and condom use has been reported [20]. Moreover, Podder et al. [21] noted that the combined use of male circumcision and ARVs is more effective in reducing disease morbidity and mortality due to HIV than the combined use of male circumcision and condoms for moderate condom compliance. However, a mathematical model to study the impact of male circumcision and condoms and microbicides use in the absence of antiretrovirals is still lacking.

The development of mathematical models has been very useful in our understanding of the dynamics of infectious diseases [22]. The literature abounds with models assessing the impact of single control strategies or in combination with antiretroviral therapy (ART). But, since only 5.2 million (34.6\%) out of 15 million people (65.4\%) living with HIV who are eligible for treatment under the new WHO guidelines are still in need [1], it is imperative to investigate the concurrent effects of other important potential and less costly alternatives such as male circumcision and condoms and microbicides use.

The rest of this paper is organized as follows. The basic and extended models are formulated and analyzed in Section 2. In Section 3, key-model-dependent quantities parameters values are provided. The impact of changes in disease thresholds (reproduction numbers) and time series of the disease dynamics are explored numerically. Conclusion follows in Section 4.

\section{Materials and Methods}

Model Formulation. Both males and females use condoms, while microbicides and circumcision are gender specific. The population under consideration is heterosexual. The female and male populations are each partitioned into three subpopulations, representing the stages of the disease: susceptibles, infectives (individuals who are infected with the virus but have not yet developed AIDS symptoms), and AIDS cases (individuals who have developed full blown AIDS and exhibit specific clinical features, herein assumed to be sexually inactive).

The female susceptibles, infectives, and AIDS cases are denoted by $S_{f}, I_{f}$, and $A_{f}$, respectively. The male susceptibles and infectives are classified into two groups: uncircumcised and circumcised denoted by $S_{m u}$ and $S_{m c}$ for susceptibles and $I_{m u}$ and $I_{m c}$ for the infectives, respectively. AIDS cases from uncircumcised and circumcised males are assumed to be sexually inactive and thus are not distinguished.

It is assumed that, at any time, recruits enter the heterosexually active population at a rate $\Lambda$. A proportion $\sigma$ of these individuals are assumed to be male susceptibles and the complementary proportion $(1-\sigma)$ are female susceptibles.
A proportion $\pi$ of male susceptibles are uncircumcised and move to the class of uncircumcised susceptibles $S_{m u}$ and the complementary proportion $(1-\pi)$ is circumcised and move to the circumcised class $S_{m c}$. Uncircumcised male $S_{m u}$ are circumcised at a rate $\phi$ and will moves to the class of circumcised male $S_{m c}$.

Female susceptibles acquire infection at a rate $\lambda_{f}$. Uncircumcised male susceptibles acquire infection at a rate $\lambda_{m}$ and circumcised male susceptibles acquire infection at a rate $\alpha \lambda_{f}$, where $\alpha$ accounts for the effect of male circumcision (partial protection). The parameter $\alpha=1-\epsilon_{s}$, where $\epsilon_{s}$ is the male circumcision efficacy.

Upon becoming infected with HIV, female susceptibles enter the class $I_{f}$ and the uncircumcised and circumcised males enter the classes $I_{m u}$ and $I_{m c}$, respectively. There is a constant emigration rate $\psi>0$ of individuals to other countries except for the AIDS patients. Infected female individuals progress to the AIDS stage at a rate $\nu$. The natural death rate $\mu$ is assumed to be proportional to the number in each class. AIDS patients have an additional disease-induced mortality rate $\rho>0$.

The infection rate $\lambda_{i}, i=f, m$, depends on the probability of transmission per partnership $\left(\beta_{i}\right)$, the rate at which an individual acquires new sexual partners per unit time $\left(C_{i}\right)$, and the proportion of infected individuals in each category $\left(I_{f}, I_{m u}, I_{m c}\right)$. The probability that an infected female will infect her male sexual partner is $\beta_{f}$ and the probability that an infected male infects his female sexual partner is $\beta_{m}$. The relative risk of infectiousness of circumcised to uncircumcised males is modeled by the parameter $\theta<1$; thus the probability of HIV transmission from an individual in category $I_{m c}$ to a susceptible in category $S_{f}$ is adjusted by the degree of infectiousness $\theta$. The model structure is depicted in Figure 1. Variables and parameters are described in Tables 1 and 2, respectively.

Putting the previous formulations and assumptions together gives the following system of nonlinear ordinary differential equations:

$$
\begin{aligned}
& S_{m u}^{\prime}=\Lambda \sigma \pi-\lambda_{f} S_{m u}-(\phi+\mu+\psi) S_{m u}, \\
& S_{m c}^{\prime}=\Lambda \sigma(1-\pi)+\phi S_{m u}-\lambda_{f} \alpha S_{m c}-(\mu+\psi) S_{m c}, \\
& S_{f}^{\prime}=\Lambda(1-\sigma)-\lambda_{m} S_{f}-(\mu+\psi) S_{f}, \\
& I_{m u}^{\prime}=\lambda_{f} S_{m u}-(v+\mu+\psi) I_{m u}, \\
& I_{m c}^{\prime}=\alpha \lambda_{f} S_{m c}-(v+\mu+\psi) I_{m c}, \\
& I_{f}^{\prime}=\lambda_{m} S_{f}-(v+\mu+\psi) I_{f}, \\
& A_{m}^{\prime}=v I_{m u}+v I_{m c}-(\rho+\mu) A_{m}, \\
& A_{f}^{\prime}=v I_{f}-(\rho+\mu) A_{f},
\end{aligned}
$$

where

$$
\lambda_{f}=\frac{\beta_{f} C_{m} I_{f}}{N_{f}}
$$


TABLe 1: Description of the models variables.

\begin{tabular}{llc}
\hline Variables & Description & Initial values \\
\hline$S_{m u}$ & Uncircumcised susceptible males & 250 \\
$S_{m c}$ & Circumcised susceptible males & 70 \\
$S_{f}$ & Susceptible females & 350 \\
$I_{m u}$ & Uncircumcised infected males & 5 \\
$I_{m c}$ & Circumcised infected males & 5 \\
$I_{f}$ & Infected females & 10 \\
$A_{m}$ & AIDS male individuals & 5 \\
$A_{f}$ & AIDS female individuals & 5 \\
\hline
\end{tabular}

TABLE 2: Models parameters description.

\begin{tabular}{llcc}
\hline Parameters & Description & Values & Source \\
\hline$\alpha$ & circumcision degree of protection & 0.896 & assumed \\
$\theta$ & reduction in infectiousness & 0.94 & assumed \\
$\phi$ & male circumcision rate & 0.09 & assumed \\
$\mu$ & natural death rate & 0.037 & Breban et al. [16] \\
$\psi$ & emigration rate & 0.01 per year & assumed \\
$v$ & progression rate to AIDS & 0.1 & Perelson and Nelson (1999) \\
$\rho$ & disease-induced mortality rate & 0.102 & Brian et al. [16] \\
$\sigma$ & proportion of male recruits & 0.5 & assumed \\
$\Lambda$ & recruitment rate & 0.03 & assumed \\
$\pi$ & proportion of uncircumcised recruits & 0.85 & assumed \\
$\epsilon_{s}$ & circumcision efficacy & 0.6 & Auvert et al. [7] \\
$\beta_{f}$ & probability of transmission for females & 0.039 & assumed \\
$\beta_{m}$ & probability of transmission for males & 0.2 & assumed \\
$C_{m}, C_{f}$ & partner acquisition rate & $3-5$ partners/year & assumed \\
$\omega_{f}$ & effectiveness of microbicide & $0.5-0.89$ & assumed \\
$\gamma_{f m}$ & condom effectiveness & 0.82 & Pinkerton and Abramson [6] \\
\hline
\end{tabular}

denotes the force of infection for female and $N_{f}=S_{f}+I_{f}$ is the total sexually active female population. Also, the force of infection for male is

$$
\lambda_{m}=\frac{\beta_{m} C_{f}\left(I_{m u}+\theta I_{m c}\right)}{N_{m}},
$$

where $N_{m}=S_{m u}+S_{m c}+I_{m u}+I_{m c}$ is the total sexually active male population (excluding AIDS patients). The total variable population size is $N_{T}=N_{f}+N_{m}+A_{f}+A_{m}$. Parameters describing sexual contact rates between men and women are constrained to obey the following relation $C_{m} N_{m}=C_{f} N_{f}$ [23].

Since the previous model (1) monitors human population, it is assumed that all the state variables and parameters are nonnegative, and all its solutions remain positive for all $t>0$. Consequently, system (1) is mathematically and epidemiologically well posed in

$$
\begin{gathered}
\Omega=\left\{\left(S_{m u}, S_{m c}, I_{m u}, I_{m c}, S_{f}, I_{f}, A_{m}, A_{f}\right) \in \mathbb{R}_{+}^{8}:\right. \\
\left.\frac{\Lambda}{\mu+\rho} \leq N_{T} \leq \frac{\Lambda}{\mu+\psi}\right\},
\end{gathered}
$$

which is positively invariant and attracting under the flow generated by the model system (1). It can be shown using the theory of permanence [24] that all solutions starting on the boundary of $\Omega$ eventually enter the interior of $\Omega$ and remain there. Therefore, the usual existence, uniqueness, and continuation results hold for system (1) in $\Omega$.

\section{Results}

3.1. Analysis of the Basic Model. In the absence of male circumcision $(\alpha=0=\phi=\theta)$, system (1) reduces to

$$
\begin{aligned}
& S_{m u}^{\prime}=\Lambda \sigma \pi-\lambda_{f} S_{m u}-(\mu+\psi) S_{m u}, \\
& S_{f}^{\prime}=\Lambda(1-\sigma)-\lambda_{m} S_{f}-(\mu+\psi) S_{f}, \\
& I_{m u}^{\prime}=\lambda_{f} S_{m u}-(v+\mu+\psi) I_{m u}, \\
& I_{f}^{\prime}=\lambda_{m} S_{f}-(v+\mu+\psi) I_{f}, \\
& A_{m}^{\prime}=v I_{m u}-(\rho+\mu) A_{m}, \\
& A_{f}^{\prime}=v I_{f}-(\rho+\mu) A_{f},
\end{aligned}
$$

where the forces of infections for females $\lambda_{f}$ and males $\lambda_{m}$ are given, respectively, by (2) and (3). The basic model (5) is defined in the set $\Psi=\left\{\left(S_{m u}, I_{m u}, S_{f}, I_{f}, A_{m}, A_{f}\right) \in \mathbb{R}_{+}^{6}\right.$ : $\left.\Lambda /(\mu+\rho) \leq N_{T} \leq \Lambda /(\mu+\psi)\right\}$. Next, we determine the asymptotic behavior of the solutions which is usually based 


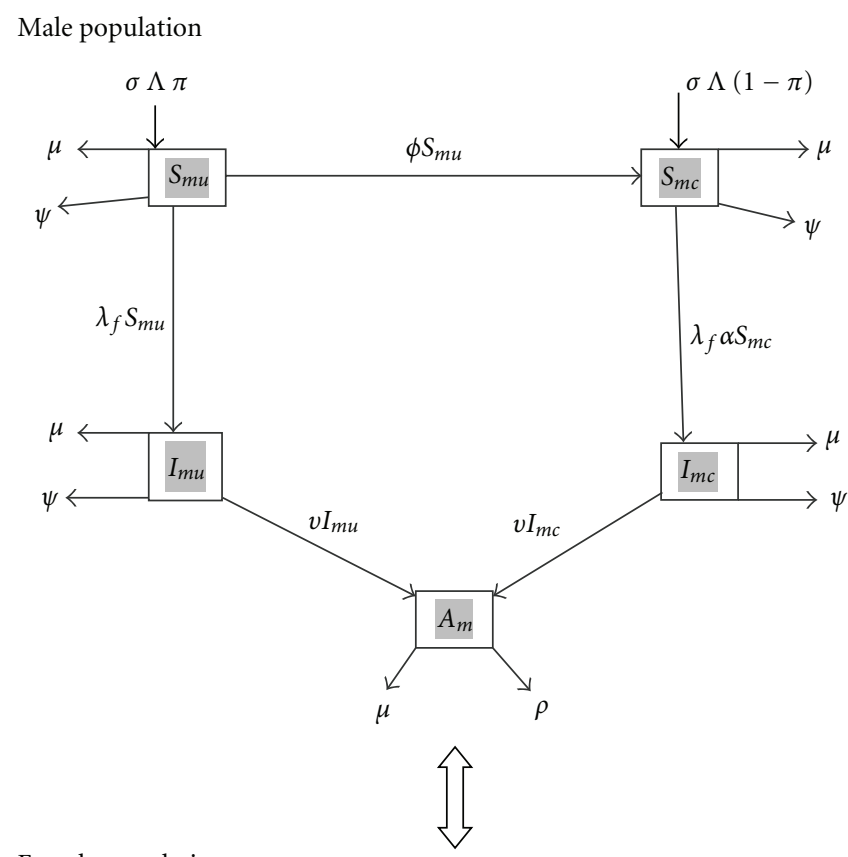

Female population

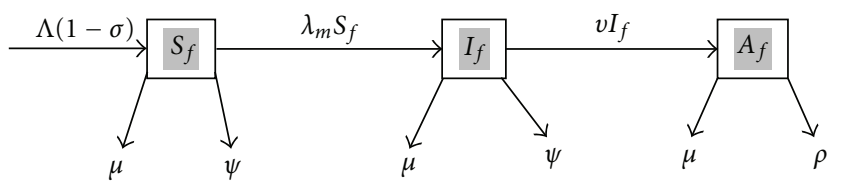

Note: the arrow $(\longrightarrow \longrightarrow$ ) denotes sexual interaction of the male and female population

Figure 1: Model compartments and flow.

on the stability of the associated equilibria. The local stability of the disease-free equilibrium (DFE) is determined based on a threshold parameter, known as the basic reproductive number.

Stability of the DFE. The DFE of model (5) is given by

$$
\begin{aligned}
\mathcal{E}^{0} & =\left(S_{m u}, S_{f}, I_{m u}, I_{f}, A_{m}, A_{f}\right) \\
& =\left(\frac{\sigma \Lambda \pi}{\mu+\psi}, \frac{\Lambda(1-\sigma)}{\mu+\psi}, 0,0,0,0\right) .
\end{aligned}
$$

Using the next generation operator method as described by Van den Driessche and Watmougth [25] and the notation therein, we compute the basic reproduction number denoted by $\mathcal{R}_{0}$. First, we need to find the rate of appearance of new infections $\mathcal{F}_{i}$, the rate of transfer of individuals out of the compartment $\mathcal{V}^{-}$, and the rate of transfer of individuals into the compartment $\mathcal{V}^{+}\left(\mathcal{V}_{i}=\mathcal{V}_{i}^{-}-\mathcal{V}_{i}^{+}\right)$:

$$
\mathcal{F}_{i}=\left(\begin{array}{c}
\frac{\beta_{f} C_{m} I_{f}}{N_{f}} S_{m u} \\
\frac{\beta_{m} C_{f} I_{m u}}{N_{m}} S_{f} \\
0 \\
0
\end{array}\right),
$$

$$
\mathcal{V}_{i}=\left(\begin{array}{c}
(v+\mu+\psi) I_{m u}-\frac{\beta_{f} C_{m} I_{f}}{N_{f}} S_{m u} \\
(v+\mu+\psi) I_{f}-\frac{\beta_{m} C_{f} I_{m u}}{N_{m}} S_{f} \\
(\rho+\mu) A_{m}-v I_{m u} \\
(\rho+\mu) A_{f}-v I_{f} c
\end{array}\right) .
$$

Their respective associated community matrices (or Jacobian) obtained by linearization at the DFE are

$$
\begin{aligned}
& \mathcal{F}=\left(\begin{array}{cc}
0 & \frac{\beta_{f} C_{m}}{N_{f}^{0}} S_{m u}^{0} \\
\frac{\beta_{m} C_{f}}{N_{m}^{0}} S_{f}^{0} & 0
\end{array}\right), \\
& \mathcal{V}=\left(\begin{array}{cc}
v+\mu+\psi & 0 \\
0 & v+\mu+\psi
\end{array}\right) .
\end{aligned}
$$

Therefore,

$$
\mathscr{F} \mathcal{V}^{-1}=\left(\begin{array}{cc}
0 & \frac{\beta_{f} C_{m}}{(v+\mu+\psi) N_{f}^{0}} S_{m u}^{0} \\
\frac{\beta_{m} C_{f}}{(v+\mu+\psi) N_{m}^{0}} S_{f}^{0} & 0
\end{array}\right) .
$$


Its largest eigenvalue is

$$
\mathcal{R}_{0}=\rho\left(\mathcal{F V}^{-1}\right)=\sqrt{\frac{\beta_{f} \beta_{m} C_{f} C_{m}}{(\mu+v+\psi)^{2}}} .
$$

$\rho$ denotes the spectral radius or largest eigenvalue [26]. From Theorem 4 of van den Driesche and Watmough [25], the following result holds.

Lemma 1. The DFE of the model (5) is locally asymptotically stable if $\mathcal{R}_{0}<1$ and unstable if $\mathcal{R}_{0}>1$.

$\mathcal{R}_{0}$, which is the threshold quantity for disease control, defines the number of new infections generated by a simple infected individual in a completely susceptible population [27].

Theorem 2. The DFE of the model (5) is globally asymptotically stable whenever $\mathcal{R}_{0}<1$.

Proof. The proof is based on using a comparison theorem [28]. The equations for the infected components in (5) can be written in terms of

$$
\begin{aligned}
\left(\begin{array}{c}
\frac{d I_{m u}(t)}{d t} \\
\frac{d I_{f}(t)}{d t}
\end{array}\right)= & (\mathcal{F}-\mathcal{V})\left(\begin{array}{c}
I_{m u}(t) \\
I_{f}(t)
\end{array}\right)-M_{1} Q_{1}\left(\begin{array}{c}
I_{m u}(t) \\
I_{f}(t)
\end{array}\right) \\
& -M_{2} Q_{2}\left(\begin{array}{c}
I_{m u}(t) \\
I_{f}(t)
\end{array}\right),
\end{aligned}
$$

where $M_{1}=1-S_{m u} / N_{m}, M_{2}=1-S_{f} / N_{f}$, and the matrices $\mathcal{F}$ and $\mathcal{V}$ are as given in (8). $Q_{1}$ and $Q_{2}$ are non-negative matrices given by

$$
Q_{1}=\left(\begin{array}{cc}
0 & \frac{\beta_{f} C_{m} S_{m u}^{*}}{N_{f}} \\
0 & 0
\end{array}\right), \quad Q_{2}=\left(\begin{array}{cc}
\frac{\beta_{f} C_{m} S_{m u}^{*}}{N_{f}} & 0 \\
0 & 0
\end{array}\right) .
$$

Thus, since $S_{m u}^{*} \leq N_{m}(t)$ and $S_{f}^{*} \leq N_{f}(t)$ in (5) for all $t \geq 0$, it follows from (11) that

$$
\left(\begin{array}{c}
\frac{d I_{m u}(t)}{d t} \\
\frac{d I_{f}(t)}{d t}
\end{array}\right) \leq(\mathcal{F}-\mathcal{V})\left(\begin{array}{c}
I_{m u}(t) \\
I_{f}(t)
\end{array}\right)
$$

Using the fact that the eigenvalues of the matrix $\mathcal{F}-\mathcal{V}$ all have negative real parts, it follows that the linearized differential inequality system (11) is stable whenever $\mathcal{R}_{0}<1$. Consequently, $\left(I_{m u}(t), I_{f}(t)\right) \rightarrow(0,0)$ as $t \rightarrow \infty$. By a comparison theorem [28], $\left(I_{m u}(t), I_{f}(t)\right) \rightarrow(0,0)$ as $t \rightarrow \infty$. Substituting $I_{m u}(t)=0=I_{f}(t)$ in the first and second equations of (5) gives $S_{m u}(t) \rightarrow S_{m u}^{*}$ and $S_{f}(t) \rightarrow S_{f}^{*}$ as $t \rightarrow \infty$. Thus, $\left(S_{m u}(t), S_{f}(t), I_{m u}(t), I_{f}(t), A_{m}(t), A_{f}(t)\right) \rightarrow$ $\left(S_{m u}^{*}, S_{f}^{*}, 0,0,0,0\right)$ as $t \rightarrow \infty$ for $\mathcal{R}_{0}<1$. Hence, the DFE $\mathcal{E}_{0}$ of (5) is globally asymptotically stable (GAS) if $\mathcal{R}_{0}<1$.

The global stability of $\mathcal{E}_{0}$ precludes the phenomenon of backward or subcritical bifurcation and a forward or transcritical bifurcation occurs. Consequently, local and

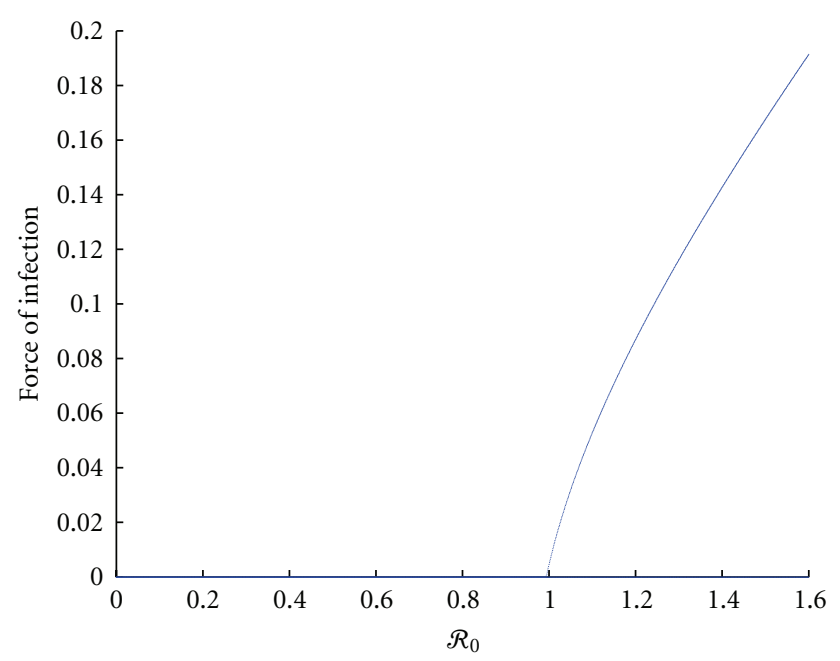

FIGURE 2: Forward bifurcation diagram for the basic model.

global stability of the endemic equilibrium of the basic model (5) follows. The forward bifurcation diagram is depicted in Figure 2.

Thus, for the basic model (without male circumcision), the HIV could be eradicated if $\mathcal{R}_{0}<1$ and will persist if $\mathcal{R}_{0}>$ 1 (for more details on disease persistence, see $[29,30]$ ).

3.2. Analysis of the Model with Male Circumcision. A proportion of the male population is circumcised. The DFE is

$$
\begin{aligned}
\mathbb{E}^{0}= & \left(S_{m u}^{0}, S_{m c}^{0}, S_{f}^{0}, I_{m u}^{0}, I_{m c}^{0}, I_{f}^{0}, A_{m}^{0}, A_{f}^{0}\right), \\
= & \left(\frac{\sigma \Lambda \pi}{\phi+\mu+\psi}, \frac{\sigma \Lambda(1-\pi)}{\mu+\psi}+\frac{\phi \sigma \Lambda \pi}{(\mu+\psi)(\phi+\mu+\psi)},\right. \\
& \left.\frac{\Lambda(1-\sigma)}{\mu+\psi}, 0,0,0,0,0\right) .
\end{aligned}
$$

For the model (1), the next generation matrix calculation [25] shows that the male-circumcision-induced reproductive number is

$$
\mathcal{R}_{s}=\sqrt{\frac{\beta_{f} \beta_{m} C_{f} C_{m}}{(\mu+v+\psi)^{2}}\left(\frac{S_{m u}^{0}+\theta \alpha S_{m c}^{0}}{S_{m u}^{0}+S_{m c}^{0}}\right)} .
$$

This threshold quantity $\left(\mathcal{R}_{s}\right)$ defines the average number of secondary cases generated by a single infected individual in a susceptible population, where a certain fraction of male individuals are circumcised. Using Theorem 2 of [25], the following result is established.

Lemma 3. The DFE of the system (1) is locally asymptotically stable if $\mathcal{R}_{s}<1$ and unstable if $\mathcal{R}_{s}>1$.

The epidemiological implication of Lemma 3 is that HIV can be eliminated from the community when $\mathcal{R}_{s}<1$, provided the initial sizes of the sub-populations of the model (1) are in the basin of attraction of $\mathcal{E}^{0}$. In other words, an influx of a small number of infected individuals into 
the population will not generate large outbreaks if the male circumcision contributes in reducing the value of $\mathcal{R}_{s}$ to below unity.

Impact of Male Circumcision. In the absence of protective effect of male circumcision $(\alpha=\theta=1)$, and $\left(\lim _{\alpha, \theta \rightarrow 1} \mathcal{R}_{s}=\right.$ $\left.\mathcal{R}_{0}\right)$. A theoretical expression for the measure of male circumcision impact can be obtained by rewriting the effective reproductive number of the model as

$$
\mathcal{R}_{s}^{2}=\mathcal{R}_{0}^{2}\left(\frac{S_{m u}^{0}}{S_{m u}^{0}+S_{m c}^{0}}\right)+\mathcal{R}_{0 s}^{2}\left(\frac{S_{m c}^{0}}{S_{m u}^{0}+S_{m c}^{0}}\right)
$$

where

$$
\mathcal{R}_{0}=\sqrt{\frac{\beta_{f} \beta_{m} C_{f} C_{m}}{(\mu+v+\psi)^{2}}}
$$

is the basic reproductive number, which measures the average number of secondary infections in the absence of male circumcision in a community and

$$
\mathcal{R}_{0 s}=\sqrt{\frac{\beta_{f} \beta_{m} C_{f} C_{m} \alpha \theta}{(\mu+v+\psi)^{2}}}
$$

is the circumcision reproductive number, which measures the average number of secondary infections when every male of the community is circumcised. Thus,

$$
\begin{aligned}
\mathcal{R}_{s}^{2}= & \mathcal{R}_{0}^{2}\left(\frac{S_{m u}^{0}}{S_{m u}^{0}+S_{m c}^{0}}\right)+\mathcal{R}_{0 s}^{2}\left(\frac{S_{m c}^{0}}{S_{m u}^{0}+S_{m c}^{0}}\right) \\
& =\mathcal{R}_{0}^{2}\left(1-\frac{S_{m c}^{0}}{S_{m u}^{0}+S_{m c}^{0}}\left(1-\frac{\mathcal{R}_{0 s}^{2}}{\mathcal{R}_{0}^{2}}\right)\right)=\mathcal{R}_{0}^{2}(1-G) .
\end{aligned}
$$

Using the notation in (Blower et al., 2002), the circumcision impact factor $G$ is given by

$$
G=\frac{S_{m c}^{0}}{S_{m u}^{0}+S_{m c}^{0}}\left(1-\frac{\mathcal{R}_{0 s}^{2}}{\mathcal{R}_{0}^{2}}\right) .
$$

It follows from (20) that if $\mathcal{R}_{0 s} \leq \mathcal{R}_{0}$, then, the circumcision impact factor $(G)$ is positive, suggesting that male circumcision has the capability of reducing the basic reproductive number and, therefore, reducing HIV morbidity and mortality in the community.

These results are summarized below.

Theorem 4. Male circumcision will have a positive impact in the community if $G>0\left(\mathcal{R}_{0 s}<\mathcal{R}_{0}\right)$, negative impact if $G<0$ $\left(\mathcal{R}_{0 s}>\mathcal{R}_{0}\right)$, and no impact if $G=0\left(\mathcal{R}_{0 s}=\mathcal{R}_{0}\right)$.

Noting that $(1-\epsilon)\left(\mathcal{R}_{0 s}^{2} / \mathcal{R}_{0}^{2}\right)<1$, for $0<\epsilon<1$, it follows (since $\left(\mathcal{R}_{0 s}^{2} / \mathcal{R}_{0}^{2}\right)<1$ ) that $G>0$ always (for any $0<\epsilon<1)$. Therefore, male circumcision has the potential to reduce secondary infections, thereby slowing the spread of HIV/AIDS (provided individuals' behavior is not compromised by the belief of full protection from male circumcision). However, this may not be the case if circumcision induces an increase in risky behavior (or risk compensation) amongst circumcised males (who believe that circumcision will provide immunity against HIV infection). In this case, the corresponding impact factor is negative, which in turn increases the associated basic reproductive number.

Existence of Backward Bifurcation. From Lemma 3, we have shown that the DFE of male circumcision model is locally asymptotically stable (LAS) if $\mathcal{R}_{s}<1$. However, this equilibrium may not be GAS in $\Omega$ for $\mathcal{R}_{s}<1$, owing to the possibility of backward bifurcation, where the stable DFE coexists with a stable endemic equilibrium when $\mathcal{R}_{s}<1$ ([31-33] and the references therein (depicted in Figure 3)). The public health implication of backward bifurcation is that the classical requirement of having the basic reproductive number less than unity, although necessary, is not sufficient for disease control. The possibility of the backward bifurcation phenomenon in (1) is investigated below. Solving the circumcision model at an arbitrary equilibrium $\mathcal{E}_{e}=$ $\left(S_{m u}^{*}, S_{m c}^{*}, S_{f}^{*}, I_{m u}^{*}, I_{m c}^{*}, I_{f}^{*}, A_{f}^{*}, A_{m}^{*}\right)$, we obtain

$$
\begin{aligned}
& 0=\sigma \Lambda \pi-\left(\lambda_{f}^{*}+\phi+\mu+\psi\right) S_{m u}^{*} \Longrightarrow S_{m u}^{*}=\frac{\sigma \Lambda \pi}{\lambda_{f}^{*}+k_{1}}, \\
& 0=\sigma \Lambda(1-\pi)+\phi S_{m u}^{*}-\left(\lambda_{f}^{*} \alpha+\mu+\psi\right) S_{m c}^{*} \\
& \Longrightarrow S_{m c}^{*}=\frac{\sigma \Lambda(1-\pi)\left(\lambda_{f}^{*}+k_{1}\right)+\phi \sigma \Lambda \pi}{\left(\lambda_{f}^{*}+k_{1}\right)\left(\alpha \lambda_{f}^{*}+k_{4}\right)} \\
& 0=\Lambda(1-\sigma)-\left(\lambda_{m}^{*}+\mu+\psi\right) S_{f}^{*} \Longrightarrow S_{f}^{*}=\frac{\Lambda(1-\sigma)}{\lambda_{m}^{*}+k_{4}} \\
& 0=\lambda_{f}^{*} S_{m u}^{*}-(v+\mu+\psi) I_{m u}^{*} \Longrightarrow I_{m u}^{*}=\frac{\sigma \Lambda \pi \lambda_{f}^{*}}{k_{2}\left(\lambda_{f}^{*}+k_{1}\right)} \\
& 0=\lambda_{f}^{*} \alpha S_{m c}^{*}-(v+\mu+\psi) I_{m c}^{*} \\
& \Longrightarrow I_{m c}^{*}=\frac{\lambda_{f}^{*} \alpha\left[\sigma \Lambda(1-\pi)\left(\lambda_{f}^{*}+k_{1}\right)+\phi \sigma \Lambda \pi\right]}{k_{2}\left(\lambda_{f}^{*}+k_{1}\right)\left(\alpha \lambda_{f}^{*}+k_{4}\right)}, \\
& 0=\lambda_{m}^{*} S_{f}^{*}-(v+\mu+\psi) I_{f}^{*} \Longrightarrow I_{f}^{*}=\frac{\lambda_{m}^{*} \Lambda(1-\sigma)}{k_{2}\left(\lambda_{m}^{*}+k_{4}\right)}, \\
& 0=v I_{m u}^{*}+v I_{m c}^{*}-(\rho+\mu) A_{m}^{*} \\
& \Longrightarrow A_{m}^{*} \\
& =\frac{\lambda_{f}^{*} \sigma \Lambda v\left[\pi\left(\lambda_{f}^{*} \alpha+k_{4}\right)+\alpha(1-\pi)\left(\lambda_{f}^{*}+k_{1}\right)+\alpha \phi \pi\right]}{k_{2} k_{3}\left(\lambda_{f}^{*}+k_{1}\right)\left(\alpha \lambda_{f}^{*}+k_{4}\right)}, \\
& 0=v I_{f}^{*}-(\rho+\mu) A_{f}^{*} \Longrightarrow A_{f}^{*}=\frac{\lambda_{m}^{*} \Lambda v(1-\sigma)}{k_{2} k_{3}\left(\lambda_{m}^{*}+k_{4}\right)},
\end{aligned}
$$




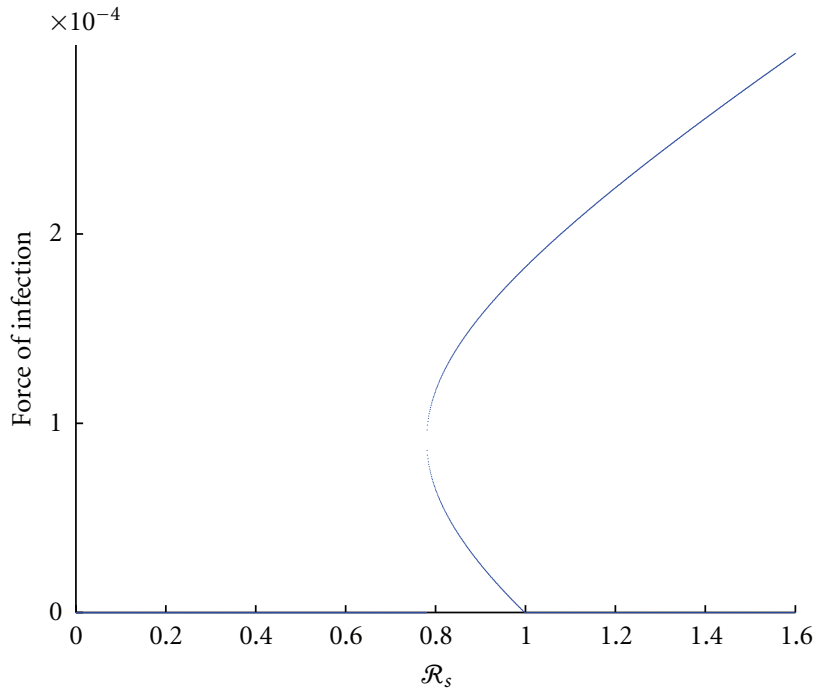

FiguRE 3: Backward bifurcation diagram for the circumcision model (1).

for $\lambda_{m}^{*}=\beta_{m} C_{f}\left(\left(I_{m u}^{*}+I_{m c}^{*} \theta\right) / N_{m}^{*}\right), \lambda_{f}^{*}=\beta_{f} C_{m}\left(I_{f}^{*} / N_{f}^{*}\right), k_{1}=$ $\phi+\mu+\psi, k_{2}=v+\mu+\psi, k_{3}=\mu+\rho, k_{4}=\mu+\psi, N_{m}^{*}=$ $S_{m u}^{*}+S_{m c}^{*}+I_{m u}^{*}+I_{m c}^{*}$, and $N_{f}^{*}=S_{f}^{*}+I_{f}^{*}$.

Therefore,

$$
\begin{aligned}
& S_{m u}^{*}=\frac{\sigma \Lambda \pi}{\left(\lambda_{f}^{*}+k_{1}\right)}, \quad S_{m c}^{*}=\frac{\sigma \Lambda(1-\pi)\left(\lambda_{f}^{*}+k_{1}\right)+\phi \sigma \Lambda \pi}{\left(\lambda_{f}^{*}+k_{1}\right)\left(\alpha \lambda_{f}^{*}+k_{4}\right)}, \\
& S_{f}^{*}=\frac{\Lambda(1-\sigma)}{\left(\lambda_{m}^{*}+k_{4}\right)}, \quad I_{m u}^{*}=\frac{\sigma \Lambda \pi \lambda_{f}^{*}}{k_{2}\left(\lambda_{f}^{*}+k_{1}\right)}, \\
& I_{m c}^{*}=\frac{\lambda_{f}^{*} \alpha\left[\sigma \Lambda(1-\pi)\left(\lambda_{f}^{*}+k_{1}\right)+\phi \sigma \Lambda \pi\right]}{k_{2}\left(\lambda_{f}^{*}+k_{1}\right)\left(\alpha \lambda_{f}^{*}+k_{4}\right)}, \\
& I_{f}^{*}=\frac{\lambda_{m}^{*} \Lambda(1-\sigma)}{k_{2}\left(\lambda_{m}^{*}+k_{4}\right)}, \\
& A_{m}^{*}=\frac{\lambda_{f}^{*} \sigma \Lambda v\left[\pi\left(\lambda_{f}^{*} \alpha+k_{4}\right)+\alpha(1-\pi)\left(\lambda_{f}^{*}+k_{1}\right)+\alpha \phi \pi\right]}{k_{2} k_{3}\left(\lambda_{f}^{*}+k_{1}\right)\left(\alpha \lambda_{f}^{*}+k_{4}\right)} \\
& A_{f}^{*}=\frac{\lambda_{m}^{*} \Lambda v(1-\sigma)}{k_{2} k_{3}\left(\lambda_{m}^{*}+k_{4}\right)},
\end{aligned}
$$

where $\lambda_{m}^{*}=\beta_{m} C_{f}\left(\left(I_{m u}^{*}+I_{m c}^{*} \theta\right) /\left(S_{m u}^{*}+S_{m c}^{*}+I_{m u}^{*}+\right.\right.$ $\left.\left.I_{m c}^{*}\right)\right), \lambda_{f}^{*}=\beta_{f} C_{m}\left(I_{f}^{*} /\left(S_{f}^{*}+I_{f}^{*}\right)\right)$. Substituting all this into $\lambda_{m}^{*}=\beta_{m} C_{f}\left(\left(I_{m u}^{*}+I_{m c}^{*} \theta\right) /\left(S_{m u}^{*}+S_{m c}^{*}+I_{m u}^{*}+I_{m c}^{*}\right)\right)$ shows that the endemic equilibria of male circumcision model satisfy the following polynomial in terms of $\lambda_{m}^{*}$ given by

$$
\lambda_{m}^{*}\left[b_{0} \lambda_{m}^{* 2}+b_{1} \lambda_{m}^{*}+b_{2}\right]=0,
$$

where

$$
\begin{aligned}
b_{0}= & \alpha \sigma \beta_{f}^{2} C_{m}^{2}+\sigma \Lambda \beta_{f} C_{m} \\
& (\pi \alpha v-\pi v+v+\mu+\psi+\alpha \phi+\alpha \mu+\alpha \psi) \\
& +\sigma \Lambda(v+\mu+\psi)(\phi+\mu+\psi),
\end{aligned}
$$

$$
\begin{aligned}
b_{1}= & \sigma \Lambda \beta_{f} C_{m}(v+\mu+\psi) \\
& (\pi \alpha v-\pi v+v+\mu+\psi+\alpha \phi+\alpha \mu+\alpha \psi) \\
& +2 \sigma \Lambda(v+\mu+\psi)^{2}(\phi+\mu+\psi) \\
& -\sigma \Lambda \alpha \beta_{m} C_{f} \beta_{f}^{2} C_{m}^{2}(\pi+\theta-\pi \theta) \\
& -\sigma \Lambda \beta_{m} \beta_{f} C_{m} C_{f}(\pi \mu+\pi \psi+\alpha \phi \theta \pi \\
& \quad+(\alpha \theta-\alpha \theta \pi)(\phi+\mu+\psi)), \\
b_{2}= & (v+\mu+\psi)^{3}(\sigma \Lambda \phi+\sigma \Lambda \mu+\sigma \Lambda \psi) \\
& -\sigma \Lambda \beta_{f} \beta_{m} C_{f} C_{m}(v+\mu+\psi) \\
& \times(\pi \mu+\pi \psi+\alpha \theta \phi+\alpha \theta \mu+\alpha \theta \psi-\alpha \theta \pi \mu-\alpha \theta \pi \psi) .
\end{aligned}
$$

The root $\lambda_{m}^{*}=0$ of (23) corresponds to the DFE (whose stability has already been established). For backward bifurcation to occur, multiple nonzero (endemic) equilibria must exist. It follows from (23) that the non-zero equilibria of the model satisfy

$$
f\left(\lambda_{m}^{*}\right)=b_{0}\left(\lambda_{m}^{*}\right)^{2}+b_{1} \lambda_{m}^{*}+b_{2}=0
$$

so that the quadratic equation (25) can be analyzed for the possibility of multiple equilibria. It is worth noting that the coefficient $b_{0}$ is always positive and $b_{2}$ is positive if $\mathcal{R}_{s}$ is less than unity, and negative if $\mathcal{R}_{s}$ is greater than unity. Hence, we have the following result.

Theorem 5. The circumcision model (5) has

(i) precisely one unique endemic equilibrium if $b_{2}<0 \Leftrightarrow$ $\mathcal{R}_{s}>1$,

(ii) precisely one unique endemic equilibrium if $b_{1}<0$ and $b_{2}=0$ or $b_{2}^{2}-4 b_{0} b_{2}=0$,

(iii) precisely two endemic equilibria if $b_{2}>0, b_{1}<0$, and $b_{1}^{2}-4 b_{0} b_{2}>0$

(iv) no endemic equilibrium otherwise.

Case (iii) indicates the possibility of backward bifurcation in the model (1) when $\mathcal{R}_{s}<1$. To find the backward bifurcation when $\mathcal{R}_{s}<1$, we set the discriminant $b_{1}^{2}-$ $4 b_{0} b_{2}$ to zero and solve for the critical value of $\mathcal{R}_{s}$. Upon computation, we obtain

$$
\mathcal{R}_{s}^{c}=\sqrt{1-\frac{b_{1}^{2}}{4 b_{0} \sigma \Lambda(\phi+\mu+\psi)(v+\mu+\psi)^{3}}},
$$

for which backward bifurcation occurs for value of $\mathcal{R}_{s}$ such that $\mathcal{R}_{s}^{c}<\mathcal{R}_{s}<1$ [33]. This phenomenon is illustrated by simulating the circumcision model system with the following set of parameter values (chosen arbitrarily for illustration purpose): $S_{m u}^{0}=0.02, S_{m c}^{0}=0.04, \theta=0.999, \beta_{m}=0.04$, $v=0.00078, \phi=0.008, \pi=0.09, \psi=0.0099, \mu=0.09$, $\beta_{f}=0.0998, C_{m}=C_{f}=3, \sigma=0.09, \Lambda=0.0677$, and $\alpha=0.981$. For the previous set of parameters, $\mathcal{R}_{s}=0.9921$ 
and $\mathcal{R}_{s}^{c}=0.9817$, so that $\mathcal{R}_{s}^{c}<\mathcal{R}_{s}<1$. When the force of infection is low, emigration is high resulting in susceptibles mixing with the infectives in (both circumcised and uncircumcised) without regard to disease risk. The resulting backward bifurcation diagram for the circumcision model is depicted in Figure 3.

\subsection{Circumcision Model with Condom Use and Microbicides.} The male circumcision model (1) is extended to incorporate two other interventions, namely, the use of condoms and microbicides. The objective is to determine what additional benefits (in terms of reduction in disease burden, i.e., HIV-induced morbidity and mortality) can be achieved by combining the male circumcision program with one or both of these interventions. The extended model is given by

$$
\begin{aligned}
& S_{m u}^{\prime}=\Lambda \sigma \pi-\lambda_{f} S_{m u}-(\phi+\mu+\psi) S_{m u} \\
& S_{m c}^{\prime}=\lambda \sigma(1-\pi)+\phi S_{m u}-\lambda_{f} \alpha S_{m c}-(\mu+\psi) S_{m c} \\
& S_{f}^{\prime}=\Lambda(1-\sigma) \pi-\lambda_{m} \omega_{f} S_{f}-(\mu+\psi) S_{f} \\
& I_{m u}^{\prime}=\lambda_{f} S_{m u}-(v+\mu+\psi) I_{m u} \\
& I_{m c}^{\prime}=\alpha \lambda_{f} S_{m c}-(v+\mu+\psi) I_{m c} \\
& I_{f}^{\prime}=\lambda_{m} \omega_{f} S_{f}-(v+\mu+\psi) I_{f} \\
& A_{m}^{\prime}=v I_{m u}-v I_{m c}-(\rho+\mu) A_{m} \\
& A_{f}^{\prime}=v I_{f}-(\rho+\mu) A_{f}
\end{aligned}
$$

where the forces of infection now read

$$
\lambda_{f}=\frac{\beta_{f} C_{m} \gamma_{f m} I_{f}}{N_{f}}, \quad \lambda_{m}=\frac{\beta_{m} C_{f} \omega_{f}\left(I_{m u}+\theta I_{m c}\right)}{N_{m}} .
$$

The model system (27) is well defined in

$$
\begin{gathered}
\Omega_{c m}=\left\{\left(S_{m u}, S_{m c}, S_{f}, I_{m u}, I_{m c}, I_{f}, A_{m}, A_{f}\right) \in \mathbb{R}_{+}^{8}:\right. \\
\left.\frac{\Lambda}{\mu+\rho} \leq N_{T} \leq \frac{\Lambda}{\mu+\psi}\right\} .
\end{gathered}
$$

In modeling condom use, we consider the use of both male and female condoms based on efficacy and compliance and assume that they confer protection independently. The parameter $\gamma_{f m}$ measures the effectiveness of condom use in reducing the probability of HIV transmission $\beta_{i}, i=$ $f, m$. Similarly, we consider the use of microbicides for female based on its efficacy and individual's compliance. The parameter $\varepsilon$ is the microbicides efficacy and $e$ represents individual's compliance. The parameter $\omega_{f}$ measures the effectiveness (which herein includes both efficacy and compliance) of female microbicides in reducing the probability of HIV transmission.
The Local Stability of the DFE. The extended model (27) has a DFE given by

$$
\begin{aligned}
\mathcal{E}_{c m}^{0}= & \left(S_{m u}^{0}, S_{m c}^{0}, S_{f}^{0}, I_{m u}^{0}, I_{m c}^{0}, I_{f}^{0}, A_{m}^{0}, A_{f}^{0}\right) \\
= & \left(\frac{\sigma \Lambda \pi}{\phi+\mu+\psi}, \frac{\sigma \Lambda(1-\pi)(\phi+\mu+\psi)+\phi \sigma \Lambda \pi}{(\mu+\psi)(\phi+\mu+\psi)},\right. \\
& \left.\frac{\Lambda(1-\sigma)}{\mu+\psi}, 0,0,0,0,0\right) .
\end{aligned}
$$

For the model (27), the next generation matrix calculation [25] shows that the effective (male circumcision, condom use, and microbicides-induced) reproduction number is

$$
\mathcal{R}_{s c m}=\sqrt{\frac{\beta_{f} \beta_{m} C_{f} C_{m} \gamma_{f m}^{2} \omega_{f}}{(\mu+v+\psi)^{2}}\left(\frac{S_{m u}^{0}+\alpha \theta S_{m c}^{0}}{S_{m u}^{0}+S_{m c}^{0}}\right)}
$$

From Theorem 2 of van den Driessche and Watmough [25], the following result holds.

Lemma 6. The DFE of the extended model (27) is locally asymptotically stable if $\mathcal{R}_{\text {scm }}<1$ and unstable if $\mathcal{R}_{s c m}>1$.

The extended model (27) exhibits similar qualitative dynamics (e.g., bifurcation) as the basic model (1). For this reason, this property is not investigated to avoid repetition.

\subsection{Model Simulations}

The Circumcision Model. The epidemiological data for the models are given in Tables 1 and 2. From the analytical results in Section 3.2, the male circumcision model (1) shows the possibility of backward bifurcation, where the diseasefree equilibrium coexists with a stable endemic equilibrium when the effective reproductive number is less than unity (Figure 3). However, this phenomenon is driven by the force of infection and the emigration rate.

When circumcision is implemented in a community as a public health intervention, the number of infectives decreases faster than when this intervention method is not accounted for (Figure 4). In Figure $4, \ell_{0}$ represents the (prevalence of) infectives in a community with no circumcision and $\ell_{s}$ represents the proportion of infectives with male circumcision as control measure. Male circumcision could help reduce the number of secondary infections (since $\mathcal{R}_{s}<\mathcal{R}_{0}$ ), thereby slowing the spread of the epidemic (by reducing the number of infectives) see Figure 3 . Thus, male circumcision which only confers partial protection to men can help, however minimal, to stem the tide of the spread of HIV infection.

The Extended Circumcision Model. The extended model (27) is simulated with the set of parameters values in Tables 1 and 2 to further assess the effects of condom, microbicides, and circumcision concurrently as HIV- preventative strategies. The effect of the number of sexual partners on secondary 


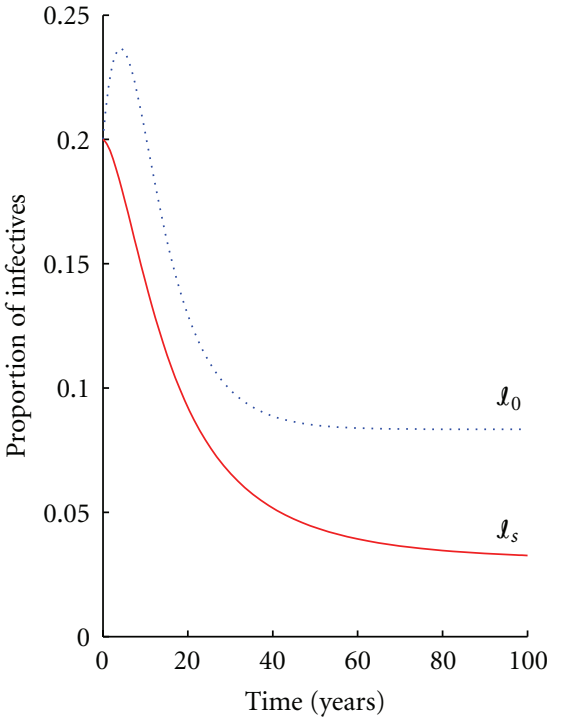

Figure 4: Dynamics of infectives with $(\phi=0.09)$ and without $(\phi=$ 0) circumcision.

transmission is depicted in Figure 5(a) $\left(\mathcal{R}_{s c m}<\mathcal{R}_{s}<\mathcal{R}_{0}\right.$ for the given parameter values). Figure 5(b) graphically depicts the effect of the various control strategies on the dynamics of the disease. When applied concurrently, male circumcision, condom and microbicides use greatly reduce the number of infectives in the community. Since this reduction does not bring the infective population to extinction, other control measures such as antiretroviral therapy are viable.

In Figure (5), $\ell_{0}$ represents the proportion of infectives in a setup with no circumcision, $l_{s}$ represents the proportion of infectives in a community where male circumcision and is common, while $\ell_{s c m}$ represents the proportion of infectives in a community with circumcision, condom and microbicides being used as preventive control measures.

\section{Conclusion}

Since its emergence in 1981, the HIV/AIDS and its associated opportunistic infections continue to pose a global public health burden. The main goal of this work was to provide a theoretical framework where male circumcision and condoms and microbicides use are implemented. The model is not country specific and could be applied to various settings, especially those where male circumcision is on the rise. While medical male circumcision and consistent condom and microbicides use hold great promise for helping to stem the tide of new HIV infections, we theoretically provide further evidence of the potential long-term population-level benefit of their combined effects by formulating a nonlinear deterministic two-sex-structured model.

Our model exhibits classical threshold behavior (or "epidemiological birth") in terms of the control reproduction number, $\mathcal{R}_{\text {scm }}$, and the basic reproduction number, $\mathcal{R}_{0}$. In the absence of antiretrovirals, microbicides and nontherapeutic measures such as male circumcision and condoms provide alternative solutions in curtailing the spread of the
HIV/AIDS epidemic. But this success is not without caveats. Under certain conditions, the model (1) undergoes backward bifurcation when the reproduction number $\mathcal{R}_{s}$ is less than unity. This phenomenon is driven by the force of infection and/or the migration rate. The epidemiological implication of such a phenomenon is that the classical requirement of having the associated reproduction number less than unity, while necessary, is not sufficient for disease control. Threshold analysis of the effective reproduction number $\mathcal{R}_{s}$ shows that the implementation of male circumcision could have positive, no, or detrimental impact depending on whether or not an impact factor, defined as $G$, is less than, equal to, or greater than zero, respectively.

The impact of these control strategies is assessed numerically by simulating the model with a reasonable set initial demographic data mostly assumed for the purpose of illustration. Numerical simulations of the models show the following Concurrent use of male circumcision, condoms, and microbicides could significantly reduce and avert more infections than when each of these control measures is used as single strategy. Thus, as male circumcision contributes in slowing down the spread of the epidemic, the infection reduction is most effective when all the three control measures are implemented concurrently $\left(\mathcal{R}_{0}>\mathcal{R}_{s}>\right.$ $\left.\mathcal{R}_{s c m}\right)$. However, they fail to completely eliminate the disease consequently, other control measures should be explored to assess the best combination prevention package, given that there is a plethora of control measures out there.

We identify some limitations of this study. The model is not structured by age, and partial reduction in the protective effect of circumcision is unaccounted for as well as antiretrovirals, information campaigns (counseling, testing), and behavioral change. We are fully aware that not considering antiretrovirals might be very unrealistic in the present state of HIV/AIDS, but this was on purpose because several studies have addressed the impact of this control strategy. Since HIV treatment does not cure the disease, but only prolongs the lifespan of infected individuals, we wished to assess the impact of similar control measures such as microbicides and condom use and male circumcision which provide some partial protection. Given that the effectiveness of a microbicides is not really known, data will be needed to estimate the lowest effectiveness of microbicides that could still have a beneficial impact. However, the main goal of this work was to provide a theoretical framework for further discussion, especially on the type of data to collect that will be helpful to inform health policy. Although the model is somehow simplistic, its provides qualitative insights on the effects the aforementioned control measures might have on the dynamics of the disease.

Our results which are driven by the model structure and its formulation are sensitive to the choice of parameter values and initial conditions. However, it is worthnoting that the proposed model captures the average characteristics of HIV combination prevention strategies and should be seen as providing general guide, rather than definitive answers or estimates, as we focused on providing insights associated with implementation of the three control strategies. Finally, we identify some limitations of this study. The model could 


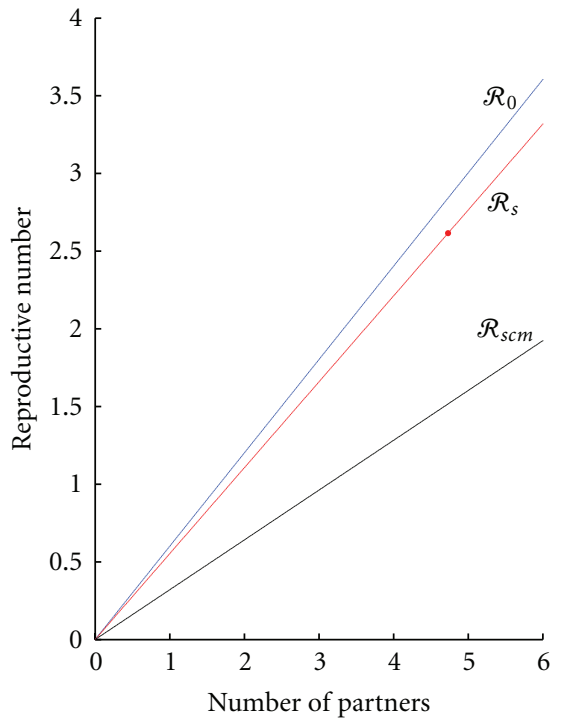

(a)

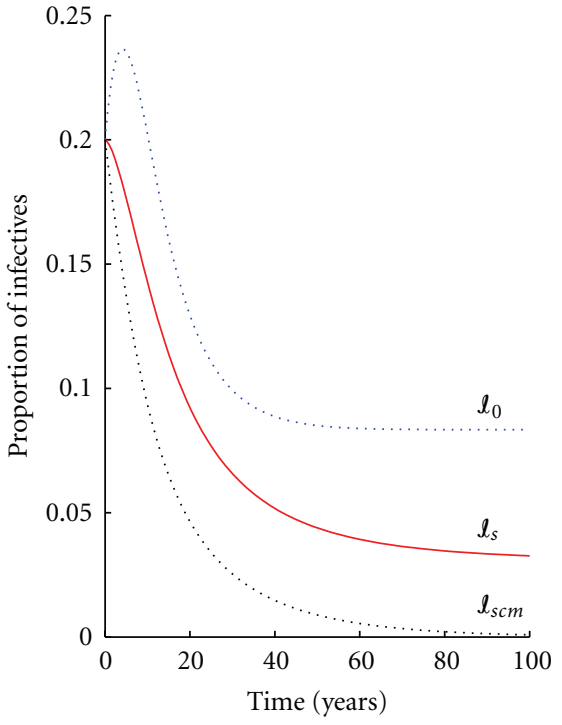

(b)

FIGURE 5: (a) Reproductive numbers for varying number of sexual partners. (b) Dynamics of infectives when there is no circumcision (dotted blue line), with circumcision (red line), and, with circumcision and condom and microbicides use (dotted black line).

be validated against field data when available. Given the model parameter uncertainty, the model parameterizations will be subjected to an extensive sensitivity analysis with adequate parameter ranges. Exploring cost-effectiveness of these combination prevention strategies is viable. How circumcision efficacy synergizes with microbicides could also be explored to find each optimum level (percentage) of effectiveness of condom and microbicides usage for maximal benefit.

\section{Summary}

Globally, HIV/AIDS has killed more than 25 million people, making it one of the most destructive epidemics in recorded history with sub-Saharan Africa carrying the highest burden. Male circumcision which provides only partial protection has been shown to reduce the risk of men acquiring HIV through female-to-male transmission by $60 \%$, while microbicides are considered one of the most promising preventive interventions to emerge over the past decade. Microbicides are $60 \%$ effective in reducing women's risk of acquiring HIV. In resource-limited settings, most women do not have the social or economic power necessary to insist on condom use and fidelity or to abandon partnerships that put them at risk. Because men often decide whether or not to use a condom during intercourse, it is generally difficult or impossible for women to ensure consistent condom use by their partners (condom acceptability is low, about $40 \%$ ). Microbicides therefore put the power of women's protection against HIV infection into their own hands as they do not require partners' cooperation. A mathematical model to study the impact of male circumcision and condoms and microbicides use in the absence of antiretroviral therapy is lacking. Although the use of antiretroviral is expanding rapidly, there are a staggering large percentage of individuals in need of treatment who are not receiving it. To this end, this study is placed in this context and is perhaps the first of its kind to assess the potential impact of these control measures in the absence of antiretrovirals. However, it is important to note that the benefit is much greater when these control strategies are used concurrently with treatment.

\section{Acknowledgments}

F. Elias acknowledges with thanks the support in part of the Dar es Salaam Institute of Technology and the Norad's Programme for Master in Mathematical Modeling at the University of Dar es Salaam.

\section{References}

[1] WHO/UNAIDS, "UNAIDS report on the global AIDS epidemic 2010," UNAIDS/10. 11E JC1958E, 2010.

[2] D. P. Wilson, P. M. Coplan, M. A. Wainberg, and S. M. Blower, "The paradoxical effects of using antiretroviral-based microbicides to control HIV epidemics," Proceedings of the National Academy of Sciences of the United States of America, vol. 105, no. 28, pp. 9835-9840, 2008.

[3] S. M. Moghadas, A. B. Gumel, R. G. McLeod, and R. Gordon, "Could condoms stop the AIDS epidemic?" Journal of Theoretical Medicine, vol. 5, no. 3-4, pp. 171-181, 2003.

[4] Zambia Report, Second Joint Annual Programme Review of National HIV/AIDS/STI/TB Intervention Strategic Plan 20022005, Government of Republic of Zambia, 2005.

[5] I. De Vincenzi, "A longitudinal study of human immunodeficiency virus transmission by heterosexual partners," The New England Journal of Medicine, vol. 331, no. 6, pp. 341-346, 1994.

[6] S. D. Pinkerton and P. R. Abramson, "Effectiveness of condoms in preventing HIV transmission," Social Science and Medicine, vol. 44, no. 9, pp. 1303-1312, 1997. 
[7] B. Auvert, D. Taljaard, E. Lagarde, J. Sobngwi-Tambekou, R. Sitta, and A. Puren, "Randomized, controlled intervention trial of male circumcision for reduction of HIV infection risk: the ANRS 1265 trial," PLoS Medicine, vol. 2, no. 11, article e298, 2005.

[8] R. C. Bailey, S. Moses, C. B. Parker et al., "Male circumcision for HIV prevention in young men in Kisumu, Kenya: a randomised controlled trial," The Lancet, vol. 369, no. 9562, pp. 643-656, 2007.

[9] A. J. Fink, "A possible explanation for heterosexual male infection with AIDS," The New England Journal of Medicine, vol. 315 , no. 18 , p. 1167, 1986.

[10] J. Hutchinson, "On the influence of circumcision on prevention of syphilis," The Medical Times and Gazette, vol. 1, pp. 542-543, 1885.

[11] S. Moses, R. C. Bailey, and A. R. Ronald, "Male circumcision: assessment of health benefits and risks," Sexually Transmitted Infections, vol. 74, no. 5, pp. 368-373, 1998.

[12] N. Siegfried, M. Muller, J. Deeks et al., "HIV and male circumcision - A systematic review with assessment of the quality of studies," The Lancet Infectious Diseases, vol. 5, no. 3, pp. 165-173, 2005.

[13] B. G. Williams, J. O. Lloyd-Smith, E. Gouws et al., "The potential impact of male circumcision on HIV in sub-Saharan Africa," PLoS Medicine, vol. 3, no. 7, article e262, 2006.

[14] R. H. Gray, G. Kigozi, D. Serwadda et al., "Male circumcision for HIV prevention in men in Rakai, Uganda: a randomised trial," The Lancet, vol. 369, no. 9562, pp. 657-666, 2007.

[15] Microbicides Trials Network, http://www.mtnstopshiv.org/ node/network.

[16] R. Breban, I. McGowan, C. Topaz, E. J. Schwartz, P. Anton, and S. Blower, "Modeling the potential impact of rectal microbicides to reduce HIV transmission in bathhouses," Mathematical Biosciences and Engineering, vol. 3, no. 3, pp. 459-466, 2006.

[17] C. Watts and P. Vickerman, "The impact of microbicides on HIV and STD transmission: model projections," AIDS, vol. 15, pp. S43-S44, 2001.

[18] R. J. Smith, E. N. Bodine, D. P. Wilson, and S. M. Blower, "Evaluating the potential impact of vaginal microbicides to reduce the risk of acquiring HIV in female sex workers," AIDS, vol. 19, no. 4, pp. 413-421, 2005.

[19] S. McCormack, "Microbicides: where are we now and what next?” HIV Therapy, vol. 4, no. 6, pp. 615-618, 2010.

[20] Z. Mukandavire, K. Bowa, and W. Garira, "Modelling circumcision and condom use as HIV/AIDS preventive control strategies," Mathematical and Computer Modelling, vol. 46, no. 11-12, pp. 1353-1372, 2007.

[21] C. N. Podder, O. Sharomi, A. B. Gumel, and S. Moses, "To cut or not to cut: a modeling approach for assessing the role of male circumcision in HIV control," Bulletin of Mathematical Biology, vol. 69, no. 8, pp. 2447-2466, 2007.

[22] R. M. Anderson and R. M. May, Infectious Diseases of Humans, Oxford University Press, Oxford, UK, 1991.

[23] C. Castillo-Chavez and S. Busenberg, "On the solution of the two-sex mixing problem," in Differential Equations Models in Biology, Epidemiology and Ecology, Proceedings, Claremont 1990, S. Busenberg and M. Martelli, Eds., vol. 92 of Lecture Notes in Biomathematics, pp. 80-98, Springer, New York, NY, USA, 1991.

[24] V. Hutson and K. Schmitt, "Permanence and the dynamics of biological systems," Mathematical Biosciences, vol. 111, no. 1, pp. 1-71, 1992.
[25] P. Van den Driessche and J. Watmough, "Reproduction numbers and sub-threshold endemic equilibria for compartmental models of disease transmission," Mathematical Biosciences, vol. 180, pp. 29-48, 2002.

[26] O. Diekmann, J. A. Heesterbeek, and J. A. Metz, "On the definition and the computation of the basic reproduction ratio $R_{0}$ in models for infectious diseases in heterogeneous populations," Journal of Mathematical Biology, vol. 28, no. 4, pp. 365-382, 1990.

[27] R. M. May and R. M. Anderson, "The transmission dynamics of Human Immunodeficiency Virus (HIV)," Philosophical Transactions of the Royal Society of London B, vol. 321, no. 1207, pp. 565-607, 1988.

[28] V. Lakshmikantham, S. Leela, and A. Martynyuk, Stability Analysis of Nonlinear Systemsand Basel, Marcel Dekker, New York, NY, USA, 1989.

[29] H. R. Thieme, "Persistence under relaxed point-dissipativity (with application to an endemic model)," SIAM Journal on Mathematical Analysis, vol. 24, no. 2, pp. 405-435, 1993.

[30] H. R. Thieme, "Uniform persistence and permanence for nonautonomous semiflows in population biology," Mathematical Biosciences, vol. 166, no. 2, pp. 173-201, 2000.

[31] J. Dushoff, W. Huang, and C. Castillo-Chavez, "Backwards bifurcations and catastrophe in simple models of fatal diseases," Journal of Mathematical Biology, vol. 36, no. 3, pp. 227248, 1998.

[32] C. M. Kribs-Zaleta and J. X. Velasco-Hernández, "A simple vaccination model with multiple endemic states," Mathematical Biosciences, vol. 164, no. 2, pp. 183-201, 2000.

[33] E. Mtisi, H. Rwezaura, and J. M. Tchuenche, "A mathematical analysis of malaria and tuberculosis co-dynamics," Discrete and Continuous Dynamical Systems B, vol. 12, no. 4, pp. 827864, 2009. 


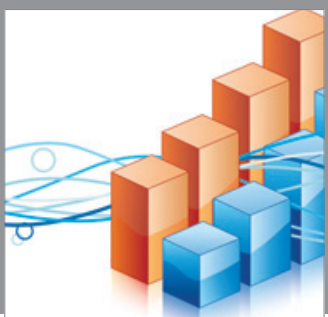

Advances in

Operations Research

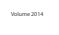

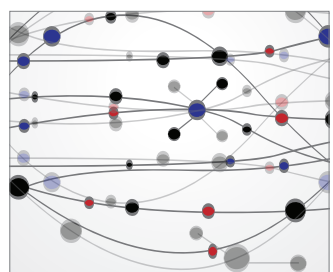

\section{The Scientific} World Journal
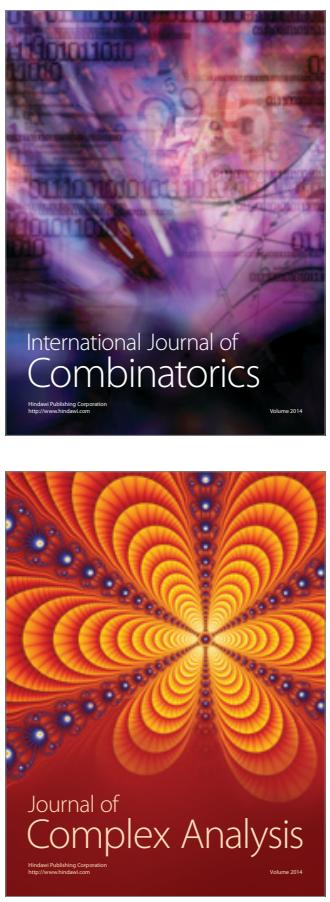

International Journal of

Mathematics and

Mathematical

Sciences
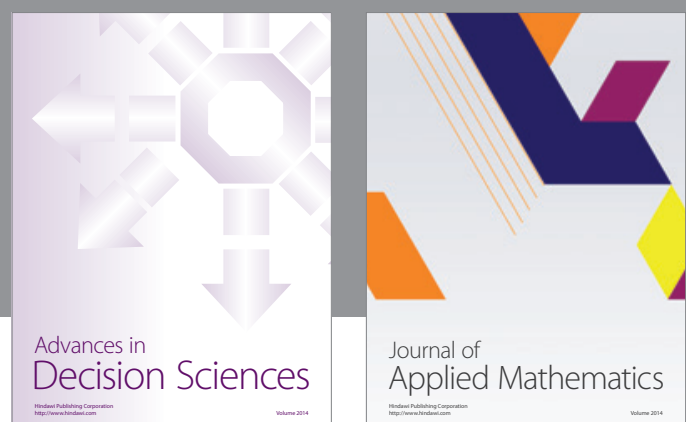

Journal of

Applied Mathematics
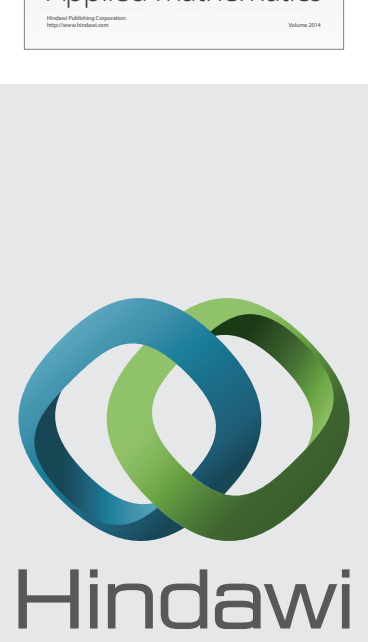

Submit your manuscripts at http://www.hindawi.com
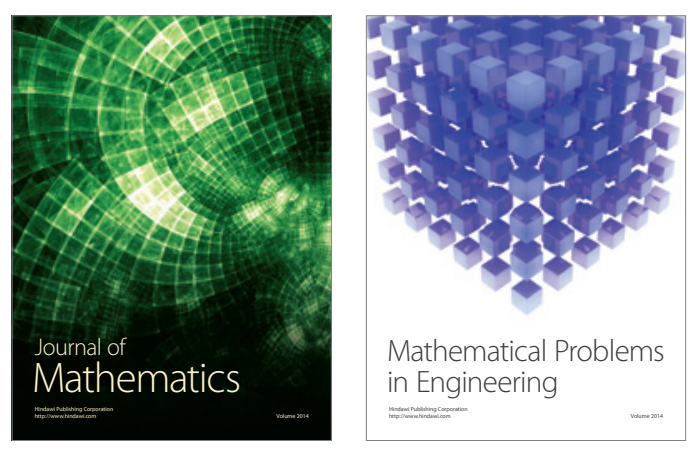

Mathematical Problems in Engineering
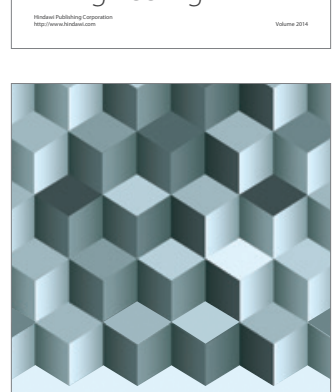

Journal of

Function Spaces
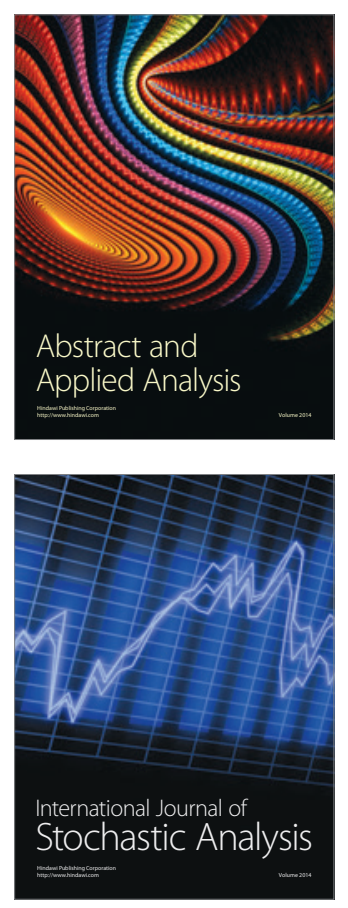

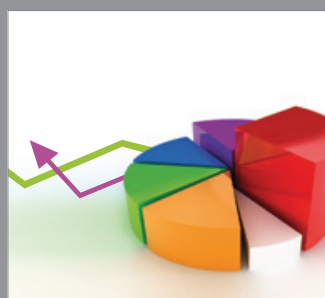

ournal of

Probability and Statistics

Promensencen
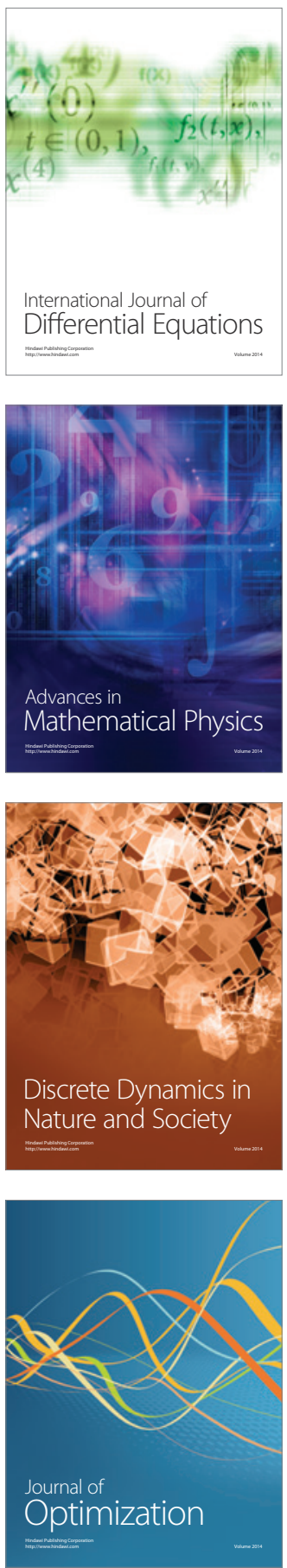